\title{
Clinical value of real-time elastography quantitative parameters in evaluating the stage of liver fibrosis and cirrhosis
}

\author{
LAN GE*, BAOMIN SHI", YE SONG, YUAN LI, SHUO WANG and XIUYAN WANG \\ Department of Ultrasound, Tongji Hospital, Tongji University, Shanghai 200065, P.R. China
}

Received July 18, 2014; Accepted March 19, 2015

DOI: $10.3892 /$ etm.2015.2628

\begin{abstract}
The aim of the present study was to assess the value of real-time elastography (RTE) quantitative parameters, namely the liver fibrosis (LF) index and the ratio of blue area (\%AREA), in evaluating the stage of liver fibrosis. RTE quantitative analysis software was used to examine 120 patients with chronic hepatitis in order to obtain the values for 12 quantitative parameters from the elastic images. The diagnostic performance of two such parameters, the LF index and \%AREA, were assessed with a receiver operating characteristic (ROC) curve to determine the optimal diagnostic cut-off values for liver cirrhosis and fibrosis. A good correlation was observed between the LF index and \%AREA with the fibrosis stage. The areas under the ROC curve for the LF index were 0.985 for the diagnosis of liver cirrhosis and 0.790 for liver fibrosis. With regard to \%AREA, the areas under the ROC curve for the diagnosis of liver cirrhosis and fibrosis were 0.963 and 0.770 , respectively. An LF index of $>3.25$ and a \%AREA of $>28.83$ for the diagnosis of cirrhosis stage resulted in sensitivity values of 100 and $100 \%$, specificity values of 88.9 and $85.9 \%$ and accuracy values of 90.8 and $88.3 \%$, respectively. The LF index and \%AREA parameters exhibited higher reliability in the diagnosis of liver cirrhosis compared with the diagnosis of the liver fibrosis stage. However, the two parameters possessed a similar efficacy in the diagnosis of liver cirrhosis and the stage of liver fibrosis. Therefore, the quantitative RTE parameters of the LF index and \%AREA may be clinically applicable as reliable indices for the early diagnosis of liver cirrhosis, without the requirement of an invasive procedure.
\end{abstract}

\section{Introduction}

Chronic viral hepatitis infection is a major cause of liver cirrhosis. The incidence rate of viral hepatitis is high in

Correspondence to: Dr Xiuyan Wang, Department of Ultrasound, Tongji Hospital, Tongji University, 389 Xincun Road, Shanghai 200065, P.R. China

E-mail: xiuyanwangcn@126.com

*Contributed equally

Key words: ultrasonography, real-time elastography, liver fibrosis
China. Precise methods for assessing the stage of liver fibrosis are required to improve prognosis, monitoring and treatment choice for patients with chronic viral hepatitis. To date, liver biopsy remains the most effective approach for the assessment of liver fibrosis. However, a biopsy is an invasive procedure, which often results in patient discomfort and bleeding, and may in certain cases lead to serious complications. In addition, the accuracy of liver biopsy may be compromised by intra- and interobserver variability and sample errors (1). Furthermore, liver biopsies are difficult to conduct repeatedly, in the cases of patients who require follow-up, due to the invasiveness of the procedure (1). Thus, numerous studies have aimed to evaluate noninvasive methods for the assessment of liver fibrosis (2). These methods include routine hematological and biochemical testing for fibrosis biomarkers, such as type IV collagen and hyaluronic acid, aspartate transaminase-to-platelet ratio index and elastographic methods, including transient elastography, acoustic radiation force impulse (ARFI), two-dimensional Shear wave elastography (2D-SWE) and real-time elastography (RTE) (2).

RTE is a relatively new method for measuring tissue elasticity, which was initially developed by Hitachi Medical Systems. In conventional ultrasound scanners, the objects under observation are physically compressed, while the echo signals of this displacement are captured and analyzed in real time. A strain image is obtained, which is color-coded according to the relative displacement, and is displayed simultaneously against a conventional 2D image. Thus, the anatomy between the tissue elasticity image and the conventional B-mode image may be determined. This technology is capable of calculating the relative stiffness of tissue (3). On the color-coded strain image, red indicates that tissue is soft, while blue indicates hard tissue, such as fibrotic tissue (3). Hence, the higher the ratio of blue area (\%AREA) the stiffer the liver parenchyma and the higher the grade of liver fibrosis. The liver fibrosis (LF) index may be subsequently quantified by performing multiple regression analysis with numerous quantitative image parameters. Previous studies have applied RTE for the diagnosis of focal lesions in breast (3), thyroid (4) and prostate gland tissues.

In 2007, Friedrich-Rust et al (5) first used RTE technology for the noninvasive assessment of liver fibrosis in patients with chronic viral hepatitis. The examinations were performed using freehand compression and the elasticity score was 
calculated using a specific formula. Initially, the intra- and interobserver variability of RTE were criticized in certain studies $(6,7)$. Freehand compression may result in liver stiffness values that differ between observers, and between separate examinations conducted by a single observer (8). Furthermore, the parameters for the assessment of liver stiffness using RTE were not previously standardized. The elasticity score, which was presented on a scale of 0-5, evaluated the degree of liver hardness using color on the RTE images; however, the scoring was subjective. RTE was observed to exhibit poor diagnostic performance in cases examining the differentiation of liver fibrosis (9). Since the absolute value of liver stiffness in RTE was variable, the 'Strain Ratio' parameter was constructed. The strain ratio was calculated using strain values from two regions of interest (ROI). A number of studies established different procedures for calculating strain ratios, which used various elastographic signals as references. Kanamoto et al (10) and Xie et al (11) used signals from the intercostal muscle as a reference, while others employed the small hepatic veins $(8,12)$ and diaphragm (13) as references. However, the strain ratio is based on a semi-quantitative technique.

Improved RTE results have been reported in numerous studies (8-18) (Table I) due to the development of heartbeat-based compression techniques, as opposed to freehand operation, and the development of software for the calculation of elasticity parameters by Hitachi Medical Systems (Tokyo, Japan). Tatsumi et al (14) first used quantitative parameters to evaluate liver fibrosis with a EUB-8500 digital ultrasound scanner (Hitachi Medical Systems). Values for nine quantitative imaging parameters were acquired, which were used to calculate the LF index with prototype quantitative analysis software. The displacement of tissue was induced by the heartbeat, as opposed to a freehand operation. In a later study, Wang et al (16) applied a similar quantitative technique using a HI VISION Preirus scanner (Hitachi Medical Systems), and obtained values for 11 parameters that characterized the stiffness of the liver parenchyma. However, the convex probe used in this study exhibited lower resolution compared with linear array probes. In addition, the method employed for calculating the elastic index with an integrative function formula was overly complicated and inconvenient for clinical application.

In the present study, the new HI VISION Avius quantitative ultrasound scanner (Hitachi Medical Systems), which included an updated version of Liver-Elasto software, was used to evaluate the liver fibrosis stage in patients with chronic hepatitis. The software automatically calculated a total of 12 quantitative parameters, of which the LF index and \%AREA were the two most important parameters for determining the degree of liver fibrosis. The present study aimed to investigate the optimal diagnosis cut-off value for the LF index and \%AREA in the differential diagnosis of liver cirrhosis and fibrosis stage, and to compare the efficacy of the two parameters.

\section{Materials and methods}

Patients. Between January 2011 and February 2013, 120 patients (male, 87 male; female, 33 ; mean age, $44.1 \pm 9$ years) with chronic hepatitis were recruited from Tongji University Hospital (Shanghai, China). The study population consisted of 111 patients with hepatitis B and nine patients with hepatitis C. All patients underwent a liver biopsy. The study criteria excluded patients with other chronic diseases that may have influenced the hepatic parenchyma and the degree of liver stiffness, including congestive heart disease, chronic renal disease, hematonosis, biliary obstructive disease and fatty liver (6). The study was conducted in accordance with the Declaration of Helsinki and with approval from the Institutional Ethics Committee of Tongji University Hospital. Written consent was obtained from all participants.

Liver histology. Following the elastography examination, liver biopsy samples were obtained from the right liver lobe via the right intercostal space under ultrasonographic guidance. Biopsy specimens were fixed in $4 \%$ formalin, and stained with hematoxylin. Liver fibrosis stages were evaluated semi-quantitatively, according to the METAVIR scoring system (19). The degree of liver fibrosis was staged on a F0-F4 scale: F0, no fibrosis; F1, portal fibrosis without septa; F2, portal fibrosis with few septa; F3, numerous septa without cirrhosis; and F4, cirrhosis. In accordance with the METAVIR scoring system, the stages of liver fibrosis were defined as non-liver fibrosis (F0), liver fibrosis (F1-F4), non-cirrhosis (F0-F3) and liver cirrhosis (F4).

RTE examination. RTE was performed using a HI VISION Avius scanner, equipped with a EUP-L52 linear probe with a central frequency of 3-7 $\mathrm{MHz}$ and quantitative analysis software. Patients were examined while in the supine position with the right arm elevated above the head. The probe was placed in the 5-8 intercostal space between the anterior axillary line and mid-axillary line, in order to display the liver parenchyma of the right anterior lobe. In this section, the right branch of the portal vein was visible, while the main portal vein was not. The probe was slightly inclined towards the direction of the heartbeat. As there were multiple reflection echoes from the liver capsule, the upper edge of the ROI (area, $2.5 \times 2.5 \mathrm{~cm}$ ) was set $1 \mathrm{~cm}$ below the liver capsule. The ROI was selected in accordance with the following guidelines: i) Avoid the main pipeline structures or vessels in the liver parenchyma; ii) avoid the rib acoustic shadow; iii) avoid sampling deep in the liver parenchyma, which may not result in clear elastic images.

Blue-green-red elastic images were formed while subjects held their breath, using the subject's heartbeat rhythm to induce the displacement of tissue. The valley of one of five stable waves was selected to form the stable static elastic image, after which quantitative analysis was initiated. An area within the ROI of $\geq 3 \mathrm{~cm}^{2}$ was selected as the analysis region. Values for the following 12 quantitative elastic imaging parameters were subsequently obtained: Mean relative strain value, standard deviation of relative strain value, \%AREA, complexity of blue area, skewness of strain histogram, kurtosis of strain histogram, contrast, entropy, inverse difference moment, angular second moment, correlation and the LF index (Fig. 1). The procedure was repeated three times and the mean value was calculated as the final result.

Definitions of the elastic image and quantitative parameters were as follows: In the 2D elastic image of the ROI, green coding represented tissue of average stiffness, red 
Table I. Devices, compression technology, elastic parameters and results of real-time elastography in previous studies.

\begin{tabular}{|c|c|c|c|c|c|}
\hline References & Patients (n) & Device & $\begin{array}{l}\text { Compression } \\
\text { method }\end{array}$ & $\begin{array}{l}\text { Elastographic } \\
\text { parameters }\end{array}$ & Results \\
\hline $\begin{array}{l}\text { Friedrich-Rust } \\
(2007)(5)\end{array}$ & 59 & EUB-8500 & Freehand & $\begin{array}{l}\text { Elasticity score } \\
\text { (calculated using } \\
\text { special formula) }\end{array}$ & $\begin{array}{l}\text { AUROC: } 0.75(F \geq F 2) \text {, } \\
0.73(F \geq F 3) \text { and } \\
0.69(F=F 4)\end{array}$ \\
\hline $\begin{array}{l}\text { Kanamoto } \\
(2009)(10)\end{array}$ & 41 & EUB-8500 & Freehand & $\begin{array}{l}\text { Elastic ratio } \\
\text { (liver parenchyma/ } \\
\text { intercostal muscle) }\end{array}$ & $\begin{array}{l}\text { AUROC: } \\
0.951(\mathrm{~F} \geq \mathrm{F} 3)\end{array}$ \\
\hline $\begin{array}{l}\text { Tatsumi } \\
(2010)(14)\end{array}$ & 44 & EUB-8500 & Heartbeat & $\begin{array}{l}\text { Liver fibrosis } \\
\text { index }\end{array}$ & $\begin{array}{l}\text { Significant differences } \\
\text { were observed between } \\
\text { F1/F2, F2/F3 and F2/F3 }\end{array}$ \\
\hline $\begin{array}{l}\text { Morikawa } \\
(2011)(15)\end{array}$ & 101 & EUB-8500 & Heartbeat & $\begin{array}{l}\text { Four image features } \\
\text { (mean, SD, area } \\
\text { and complexity) }\end{array}$ & $\begin{array}{l}\text { AUROC for cirrhosis: } \\
\text { mean, } 0.91 \text {; SD, } 0.84 ; \\
\text { area, } 0.91 \text {; and } \\
\text { complexity, } 0.93\end{array}$ \\
\hline $\begin{array}{l}\text { Koizumi } \\
(2011)(8)\end{array}$ & 70 & EUB-7500 & Heartbeat & $\begin{array}{l}\text { Elastic ratio } \\
\text { (intrahepatic vessel/ } \\
\text { liver parenchyma) }\end{array}$ & $\begin{array}{l}\text { AUROC: } 0.89(F \geq F 2) \\
0.94(F \geq F 3) \text { and } \\
0.95(F=F 4)\end{array}$ \\
\hline $\begin{array}{l}\text { Wang } \\
(2012)(16)\end{array}$ & 75 & $\begin{array}{l}\text { HI VISION } \\
\text { Preirus }\end{array}$ & Heartbeat & $\begin{array}{l}\text { Eleven parameters, } \\
\text { including } \\
\text { elastic index }\end{array}$ & $\begin{array}{l}\text { AUROC: } 0.93(F \geq F 1) \\
0.92(F \geq F 2), 0.84 \\
(F \geq F 3) \text { and } 0.66(F=F 4)\end{array}$ \\
\hline $\begin{array}{l}\text { Ochi } \\
(2012)(12)\end{array}$ & 187 & EUB-7500 & Heartbeat & $\begin{array}{l}\text { Elastic ratio } \\
\text { (intrahepatic vessel/ } \\
\text { liver parenchyma) }\end{array}$ & $\begin{array}{l}\text { Diagnostic } \\
\text { accuracy: } \\
82.6-96.0 \%\end{array}$ \\
\hline $\begin{array}{l}\text { Xie } \\
(2012)(11)\end{array}$ & 71 & HV-900 & Freehand & $\begin{array}{l}\text { Elastic ratio } \\
\text { (liver parenchyma/ } \\
\text { intercostal muscle) }\end{array}$ & $\begin{array}{l}\text { AUROC: } 0.863 \\
(\mathrm{~F} \geq \mathrm{F} 2) \text { and } \\
0.797(\mathrm{~F}=\mathrm{F} 4)\end{array}$ \\
\hline $\begin{array}{l}\text { Ferraioli } \\
(2012)(17)\end{array}$ & 130 & EUB-8500 & Heartbeat & $\begin{array}{l}\text { Liver fibrosis index } \\
\text { (calculated by } \\
\text { special formula) }\end{array}$ & $\begin{array}{l}\text { AUROC: } 0.74(F \geq F 2), \\
0.80(F \geq F 3) \text { and } \\
0.80(F=F 4)\end{array}$ \\
\hline $\begin{array}{l}\text { Yada } \\
(2013)(18)\end{array}$ & 245 & $\begin{array}{l}\text { HI VISION } \\
\text { Preirus }\end{array}$ & Heartbeat & Liver fibrosis index & $\begin{array}{l}\text { AUROC: } \\
0.865(F \geq F 3)\end{array}$ \\
\hline $\begin{array}{l}\text { Chung } \\
(2013)(9)\end{array}$ & 74 & $\begin{array}{l}\text { HI VISION } \\
\text { Preirus }\end{array}$ & $\begin{array}{l}\text { Not } \\
\text { mentioned }\end{array}$ & $\begin{array}{l}\text { Elasticity } \\
\text { score }(0-5)\end{array}$ & $\begin{array}{l}\text { AUROC: } 0.507(\mathrm{~F} \geq \mathrm{F} 2) \\
\text { and } 0.767(\mathrm{~F}=\mathrm{F} 4)\end{array}$ \\
\hline $\begin{array}{l}\text { Paparo } \\
(2013)(13)\end{array}$ & 60 & $\begin{array}{l}\text { MyLab } \\
\text { Twice }\end{array}$ & Freehand & $\begin{array}{l}\text { Elastic ratio } \\
\text { (diaphragm/liver } \\
\text { parenchyma) }\end{array}$ & $\begin{array}{l}\text { AUROC: } \\
0.86(\mathrm{~F} \geq \mathrm{F} 2) \\
\text { and } 0.909(\mathrm{~F} \geq \mathrm{F} 3)\end{array}$ \\
\hline
\end{tabular}

All devices listed were developed by Hitachi Medical Systems (Tokyo, Japan), with the exception of the MyLab Twice scanner (Esaote, S.p.A, Genoa, Italy). AUROC, area under the receiver operating characteristic curve; SD, standard deviation; F0, no fibrosis; F1, portal fibrosis without septa; F2, portal fibrosis with few septa; F3, numerous septa without cirrhosis; and F4, cirrhosis.

coding represented a lower than average stiffness and the blue coding represented harder than average stiffness. The elastic parameters were calculated automatically using Liver-Elasto software without post-processing. The \%AREA represented the relative percentage of pixels in the ROI with a lower than average deformation. The more advanced the stage of liver fibrosis, the stiffer the liver parenchyma. Therefore, the larger the relative area with lower than average deformation, the larger the blue area in the ROI (Fig. 2). The LF index was calculated using a multiple linear regression equation involving 11 parameters, which represented the degree of liver fibrosis. The higher the LF index, the greater the degree of liver fibrosis.
Statistical analysis. SPSS software, version 20.0 (IBM SPSS, Armonk, NY, USA) was used for statistical analysis. The liver biopsy result was used as the reference for diagnosis comparison. Measurement data are expressed as the mean \pm standard deviation. Spearman's correlation analysis was conducted to assess the correlation between the quantitative parameters and the liver fibrosis grade. The LF index and \%AREA of the four groups (non-hepatic fibrosis, hepatic fibrosis, non-cirrhosis and liver cirrhosis stages) were analyzed for normality using the Kolmogorov-Smirnov test. Comparison of the mean values between two normal distribution groups was performed using a two-sample Student's t-test. In cases of heterogeneity of variance, the nonparametric Mann-Whitney U test was used. The 


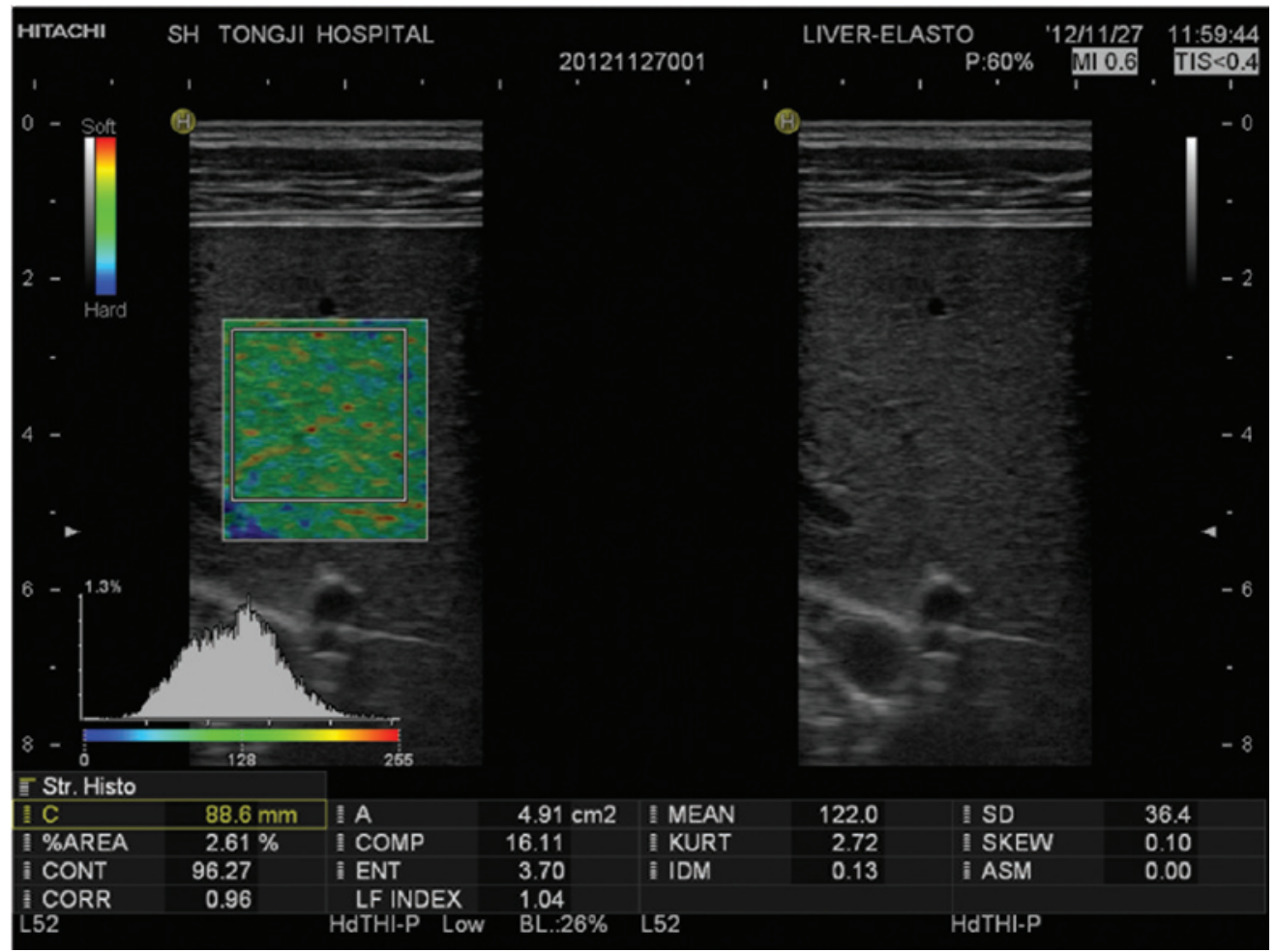

Figure 1. Elastic graph showing organization diffusion elasticity imaging and the assessment of 12 quantitative parameters.

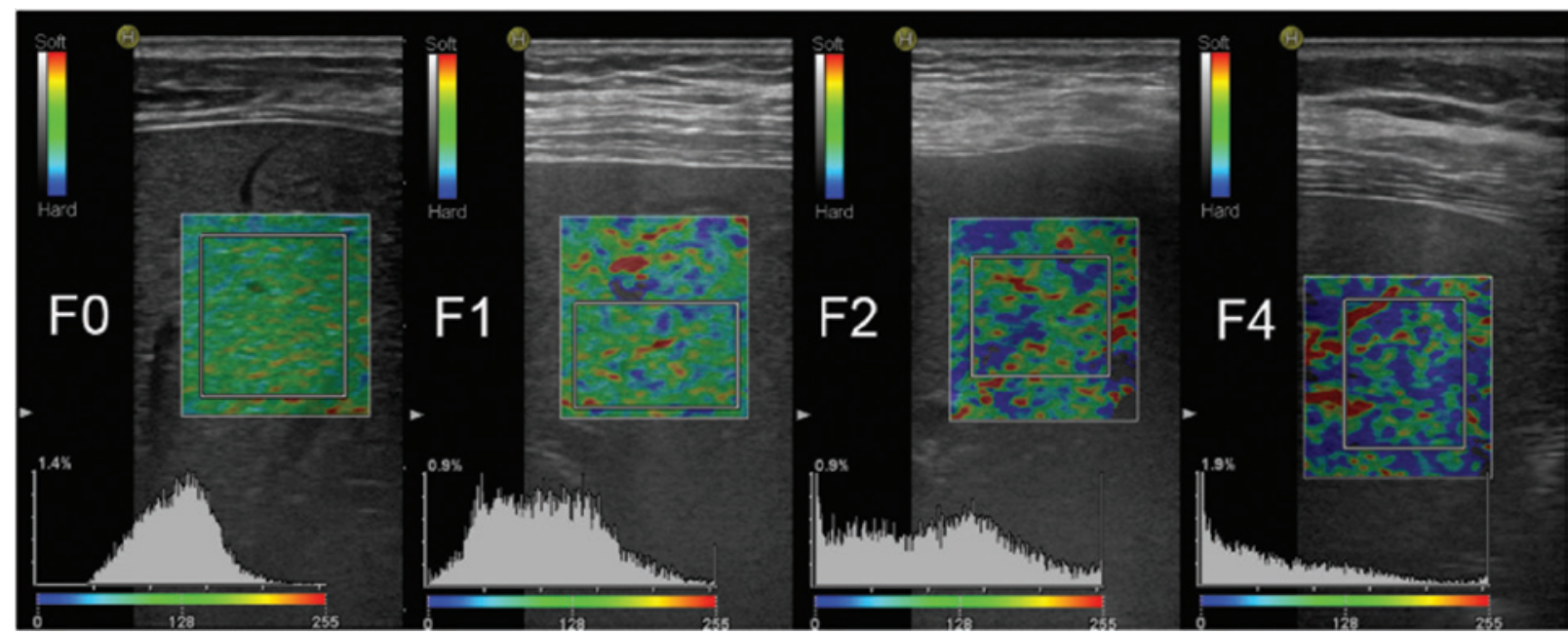

Figure 2. \%AREA in the region of interest increases with the increase in the degree of liver fibrosis. \%AREA, ratio of blue area.

diagnostic performances of the LF index and \%AREA for early cirrhosis and liver fibrosis stage were assessed using receiver operating characteristic (ROC) curves. The area under the curve (AUC) was calculated and compared using the $\mathrm{Z}$ test. The maximum Youden's index was calculated to identify the optimal cut-off value for the diagnosis of early cirrhosis and liver fibrosis, and to calculate the sensitivity, specificity, accuracy and positive predictive values. $\mathrm{P}<0.05$ was considered to indicate a statistically significant difference.

\section{Results}

Correlation analysis. Spearman's rank correlation analysis was performed using SPSS software. A good correlation was observed between the LF index and the \%AREA with the pathological stage; the correlation coefficient values were $0.711(\mathrm{P}<0.001)$ and $0.632(\mathrm{P}<0.001)$, respectively. Of the 120 patients with chronic hepatitis, 33 were classified as stage F0, 27 had stage F1, 21 were classified as stage F2, 18 had stage F3 and 21 exhibited stage F4. The statistical values of the two main elastic quantitative parameters for each liver fibrosis stage are presented in Table II.

Comparison of RTE parameters. In the no liver cirrhosis stages (F0-F3; n=99), the LF index was 2.36 \pm 0.65 , while for the early cirrhosis stage ( $F 4 ; n=21)$, the $L F$ index was $4.10 \pm 0.49$. The LF index of the two groups showed normal distribution, and statistically significant differences were observed in the 
Table II. Values of the elastic quantitative parameters for each stage of liver fibrosis.

\begin{tabular}{lcccrr}
\hline Parameters & F0 stage $(\mathrm{n}=33)$ & F1 stage $(\mathrm{n}=27)$ & F2 stage $(\mathrm{n}=21)$ & F3 stage $(\mathrm{n}=18)$ & F4 stage $(\mathrm{n}=21)$ \\
\hline \%AREA & $15.20 \pm 7.97$ & $18.76 \pm 8.63$ & $23.67 \pm 13.63$ & $24.75 \pm 8.38$ & $44.42 \pm 7.35$ \\
LF index & $2.03 \pm 0.54$ & $2.26 \pm 0.53$ & $2.48 \pm 0.78$ & $2.97 \pm 0.35$ & $4.10 \pm 0.49$ \\
\hline
\end{tabular}

Results are expressed as the mean \pm standard deviation. LF index, liver fibrosis index; \%AREA, ratio of blue area.
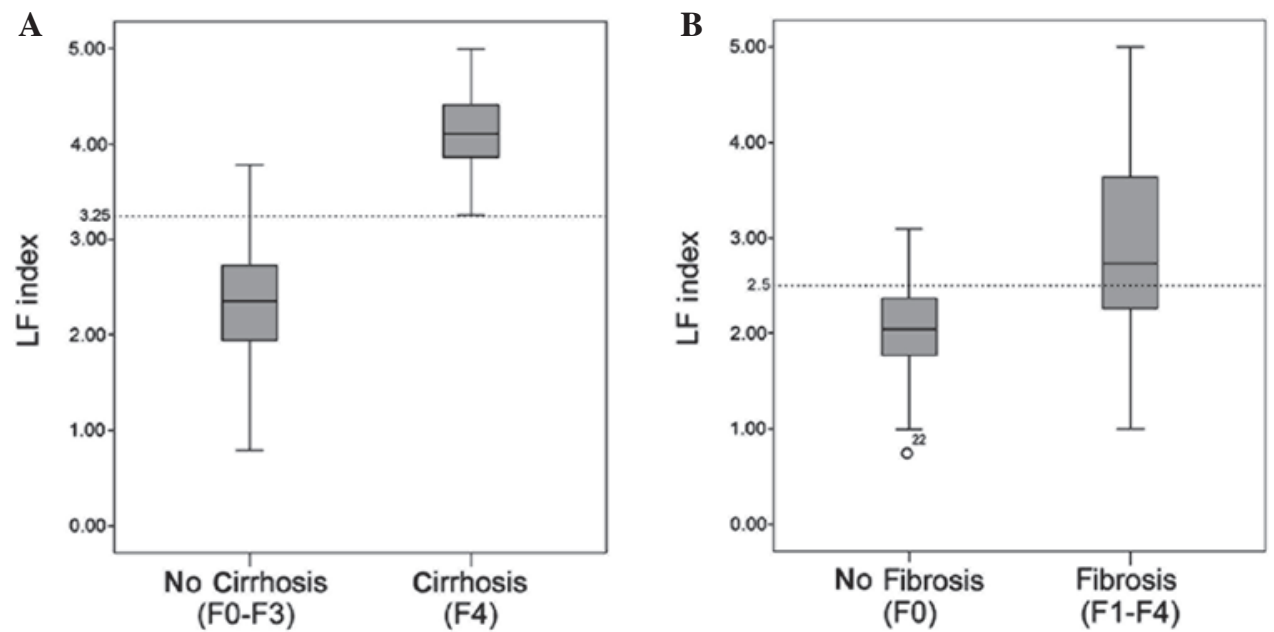

Figure 3. Box plots of the LF index revealed statistically significant differences between (A) liver cirrhosis and liver cirrhosis, and (B) liver fibrosis stage and liver fibrosis stage. LF index, liver fibrosis index.
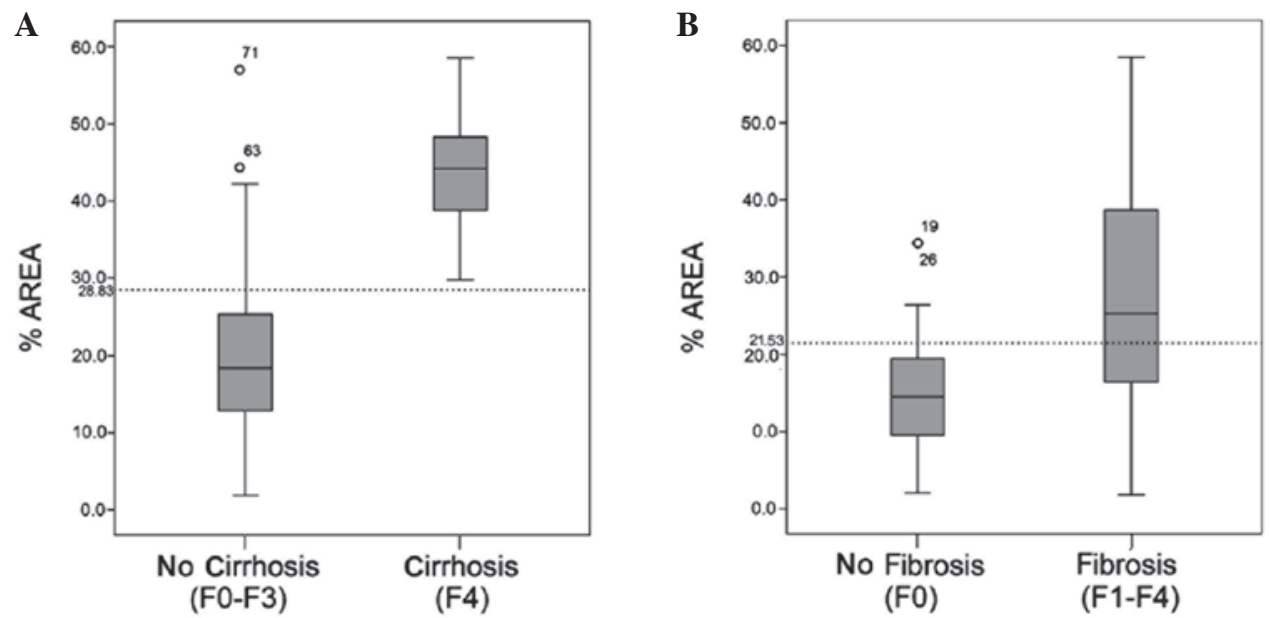

Figure 4. (A) Box plots of the \%AREA revealed statistically significant differences between (A) liver cirrhosis and liver cirrhosis, and (B) liver fibrosis stage and liver fibrosis stage. \%AREA, ratio of blue area.

LF index between the two groups $(\mathrm{P}<0.001)$. The LF index values for the no hepatic fibrosis $(\mathrm{F} 0 ; \mathrm{n}=33)$ and hepatic fibrosis stages $(\mathrm{F} 1-\mathrm{F} 4 ; \mathrm{n}=87)$ were $2.03 \pm 0.54$ and $2.91 \pm 0.90$, respectively. The LF index of the two groups exhibited normal distribution. However, due to the presence of heterogeneity of variance, two independent samples nonparametric tests and the Mann-Whitney $U$ test were performed, and statistically significant differences in LF index were identified between the two groups $(\mathrm{P}<0.001$; Fig. 3$)$.

The \%AREA values for the early liver cirrhosis $(\mathrm{F} 4 ; \mathrm{n}=21)$ and no liver cirrhosis (F0-F3; $n=99)$ groups were 19.70 \pm 10.28 and $44.42 \pm 7.35$, respectively. The \%AREA of the two groups exhibited a normal distribution and a statistically significant difference was observed in the \%AREA between the two groups $(\mathrm{P}<0.001)$. The \%AREA values for the no hepatic fibrosis $(\mathrm{F} 0 ; \mathrm{n}=33)$ and hepatic fibrosis $(\mathrm{F} 1-\mathrm{F} 4 ; \mathrm{n}=87)$ stages were $15.20 \pm 7.97$ and $27.38 \pm 13.83$, respectively. The \%AREA values of the two groups exhibited normal distribution; however, due to the presence of heterogeneity of variance, two independent samples nonparametric tests and the Mann-Whitney U test were performed. A statistically significant difference in the \%AREA was observed between the two groups $(\mathrm{P}<0.001$; Fig. 4). 


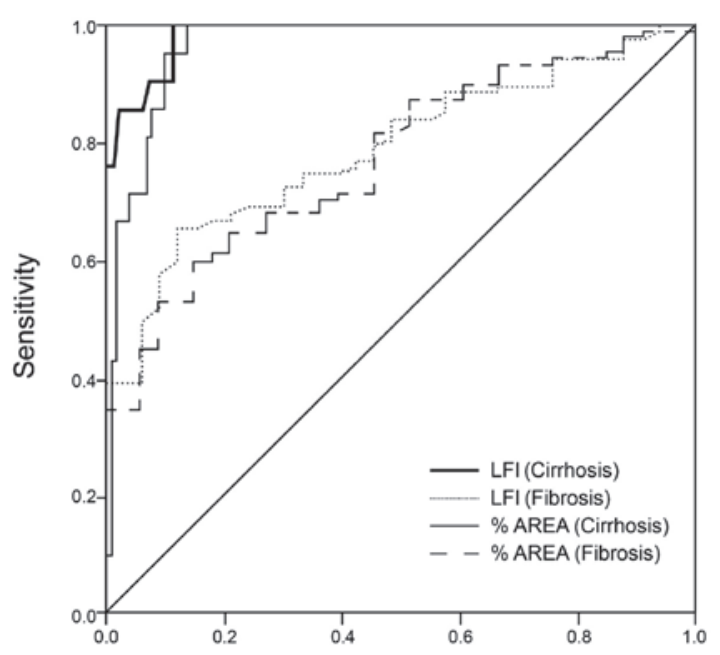

Figure 5. Receiver operating characteristic (ROC) curves of the LFI for the diagnosis of cirrhosis (bold line) and fibrosis (dotted line), and of the \%AREA for the diagnosis of cirrhosis (line) and fibrosis (broken line). The area under the ROC curves for the diagnosis of cirrhosis using the HI VISION Avius device were superior to those for the diagnosis of fibrosis. LFI, liver fibrosis index; \%AREA, ratio of blue area.

ROC curve analysis. ROC curves were produced for the LF index and \%AREA in diagnosing liver cirrhosis and fibrosis stage (Fig. 5). AUC values for the diagnosis of liver cirrhosis and fibrosis using the LF index were 0.985 and 0.790 , respectively, while using the \%AREA, the AUC values were 0.963 and 0.770 , respectively. According to the maximum Youden's index, the optimal LF index values for the diagnosis of liver cirrhosis and fibrosis stage were 3.25 and 2.5 , respectively. The sensitivity of an LF index of $>3.25$ in the diagnosis of cirrhosis stage was $100 \%$, the specificity was $88.9 \%$, the accuracy was $90.8 \%$, the positive predictive value was $65.6 \%$ and the Youden's index value was $88.9 \%$. The sensitivity of an LF index of $>2.5$ in the diagnosis of liver fibrosis stage was $65.5 \%$, the specificity was $87.9 \%$, the accuracy was $71.7 \%$, the positive predictive value was $93.4 \%$ and the Youden's index value was $53.4 \%$. According to the maximum Youden's index, the optimal \%AREA values for the diagnosis of liver cirrhosis and liver fibrosis stage were 28.83 and $21.53 \%$, respectively. The sensitivity of a \%AREA of $>28.83$ in the diagnosis of cirrhosis stage was $100 \%$, the specificity was $85.9 \%$, the accuracy was $88.3 \%$, the positive predictive value was $60 \%$ and the Youden's index value was $85.9 \%$. The sensitivity of a \%AREA of $>21.53$ in the diagnosis of liver fibrosis stage was $59.8 \%$, the specificity was $84.8 \%$, the accuracy was $66.7 \%$, the positive predictive value was $91.2 \%$ and the Youden's index value was $44.6 \%$ (Fig. 5).

The AUC values of the four groups were compared using the Z-test. No statistically significant difference was observed in the ROC curve between the LF index and \%AREA for the diagnosis of early cirrhosis $(\mathrm{Z}=1.20 ; \mathrm{P}=0.23)$. Furthermore, no statistically significant difference was identified in the ROC curve between the LF index and \%AREA for the diagnosis of liver fibrosis $(\mathrm{Z}=0.33 ; \mathrm{P}=0.74)$. These results indicated that the LF index and \%AREA parameters possess a similar efficacy in the diagnosis of early liver cirrhosis and fibrosis.

However, a statistically significant difference was observed in the AUC values for the LF index between the diagnosis of early liver cirrhosis and fibrosis $(\mathrm{Z}=4.65 ; \mathrm{P}<0.001)$. In addition, a statistically significant difference in the AUC values for the \%AREA was identified between the diagnosis of early liver cirrhosis and fibrosis $(\mathrm{Z}=4.12 ; \mathrm{P}<0.001)$. These findings indicated that the efficacy of the LF index and \%AREA was higher for the diagnosis of liver cirrhosis compared with the diagnosis of liver fibrosis.

\section{Discussion}

Numerous studies have investigated novel noninvasive methods for diagnosing liver cirrhosis and fibrosis with the potential to replace liver biopsy. Two primary noninvasive methods exist for the diagnosis the liver fibrosis. One method is based on blood serum markers or indices comprising different combinations of serum markers, such as the FibroTest ${ }^{\circledR}$ (APHP Assistance Publique, Paris, France), while the other method, elastography, is based on the measurement of tissue elasticity. Elastography was initially proposed by Ophir et al in 1991 (20) and gradually developed into a relatively mature imaging tool. Elastography techniques include transient elastography (FibroScan ${ }^{\circledR}$; Echosens, Paris, France), ARFI (Siemens AG, Munich, Germany), 2D-SWE (Aixplorer ${ }^{\circledR}$, SuperSonic Imagine (SSI), France) and RTE (Hitachi Medical Systems,).

In 2007, Frederich-Rust et al (5) reported the preliminary clinical application of RTE for the assessment of liver fibrosis. The authors established a quantitative elasticity score, calculated by assessing color-coded strain images using the Matlab computer program (MathWorks, Inc., Natick, MA, USA). Freehand compression of the probe was employed and a scale of 0-6 arbitrary units was applied for pressure measurement. However, the inter- and intraobserver variability of RTE was criticized. The freehand method of applying compression was an influential factor that may vary significantly and be difficult to standardize. Observers with different levels of experience and training may affect the results of the RTE examination by applying varying levels of freehand pressure. However, RTE technology is continuously improving and developing. For example, the compression required for generating elastic deformation of the ROI, initially induced by a freehand operation $(5,10,11)$, may now be supplied by the regular cardiovascular pulsation of the patient $(8,12,14-18)$, which reduces the subjective error inherent in the manual application of pressure. Furthermore, the elastic parameters have progressed from the initial qualitative elasticity scores $(0-5)$ (9) to a semi-quantitative strain rate ratio method $(8,10-12)$, and finally to the quantitative parameters currently available, such as the elastic index (14-18). In addition, elastic parameters are now generated using a number of static color-coded images or video clips, which are analyzed by computer software, rather than a single static image captured selectively by observers. As the examiner may intentionally select the best images from a dynamic clip, selection bias is usually high during analysis of a single static image $(5,15,21,22)$. Previous studies have demonstrated that RTE imaging is not constrained by ascites (23), distance (23) or by the position of the liver lobes (8), in addition to exhibiting good repeatability for different operators between and within groups $(8,22)$. In 2010, Tatsumi et al (14) first evaluated a prototype quantitative RTE technique using an EUB-8500 ultrasound scanner. Six RTE images were 
collected per patient and analyzed using the included software to calculate nine image parameters, including the ratio of blue area, complexity of blue area and the mean relative strain value. Multiple regression analysis was performed on the nine image parameters to quantify the LF index. In order to overcome the problems of freehand operation, newer RTE modules were designed to produce elastograms generated by the heartbeat of the patient. In the present study, a new generation RTE technique, developed by Hitachi Medical Systems, was employed. The technique utilized RTE quantitative analysis software, relying on the patient's own cardiovascular pulsation to produce compression. A total of 12 quantitative parameters were calculated automatically using the updated software integrated in the HI VISION Avius ultrasound scanner.

Previous animal models (24) and clinical studies (16-18) have suggested that the LF index is an effective parameter for evaluating the degree of liver fibrosis. The LF index has been demonstrated to correlate well with the histological grade, and may be clinically applicable for the diagnosis of liver cirrhosis and fibrosis. In the present study, the diagnostic value of the LF index and \%AREA were simultaneously evaluated. A good correlation was observed between the LF index and the stage of liver fibrosis, which was superior compared with the correlation with \%AREA. The higher the value of the \%AREA and LF index parameters, the higher the indicated degree of liver fibrosis. Whether using the LF index or the \%AREA, the values of the two groups exhibited increased overlap when discriminating between cases with and without liver fibrosis. These results suggested that the differential ability in distinguishing between patients with and without liver fibrosis was reduced. This conclusion was additionally confirmed by the size of the AUC for the two quantitative parameters (0.790 and 0.770 , respectively), which were lower than those in distinguishing between patients with or without liver cirrhosis (0.985 and 0.963 , respectively). The two quantitative parameters exhibited less overlap in distinguishing a diagnosis between those with and without cirrhosis. The specificity, sensitivity and accuracy values for the LF index and \%AREA in the diagnosis of cirrhosis stage were higher compared with those for the diagnosis of liver fibrosis, indicating that the LF index and \%AREA had a higher reliability in the diagnosis of early liver cirrhosis compared with the diagnosis of liver fibrosis. However, during the simultaneous diagnosis of early liver cirrhosis and fibrosis, no statistically significant difference was identified in the AUC between the LF index and \%AREA, which indicated that the two quantitative parameters possessed a similar efficacy.

An additional problem with assessing the efficacy of RTE parameters is that the placement of the ROI in liver elastography differs between studies. Friedrich-Rust et al (5) and Tatsumi et al (14) set the elastography ROI entirely inside the liver parenchyma. Alternative studies $(6,11)$ have criticized this placement. These studies suggest that the ROI for elastography should include the targeted liver parenchyma and the surrounding tissues, which have a mixed strain that contains soft tissue (subcutaneous adipose tissue) and harder tissue (diaphragm and intercostal muscles). Since the average strain inside the ROI is computed relatively in RTE, placement of the ROI inside the liver may lead to technical errors. Authors who suggested this hypothesis considered that the surrounding tissues possessed similar elasticity in all patients, regardless of the stage of their condition. However, in the present study, placing the ROI entirely inside the liver did not reduce the accuracy of the experiment.

However, there remain a number of factors that influence the results of RTE imaging. Firstly, the breathing cooperation of patients is crucial, and the patient is required to hold their breath during the inspection process to exclude the liver displacement caused by breathing. Certain patients exhibit poor compliance and are unable to hold their breath, which may waste time and energy, and affect the stability and analysis results of the elastic image. Secondly, the generation of the elastic images no longer depends on the operator to apply compression. Tissue compression may now be induced by the rhythmic beats of the heart on the ROI, which limits human error to a certain extent and improves the reliability and reproducibility of data. However, different patients exhibit different heart rates, which are difficult to subject to quantized control. The effects of heart rate variation on the efficacy of RTE examination and subsequent quantitative analysis require further study. Thirdly, with regard to the results of the present study, the efficacy of RTE for the differential diagnosis of liver cirrhosis and fibrosis requires confirmation in further studies with an enlarged sample population and multicentric design. In addition, the intra-observer variability of this novel technology requires further study. Therefore, future prospective studies with blinded comparisons are required to compare the efficacy of RTE with alternative elastography techniques, including transient elastography and ARFI, for the assessment of liver fibrosis

In conclusion, RTE imaging is an effective method for the noninvasive assessment of liver fibrosis. The LF index and \%AREA are two key parameters that may aid in the early clinical diagnosis of liver cirrhosis.

\section{References}

1. Sumida Y, Nakajima A and Itoh Y: Limitations of liver biopsy and non-invasive diagnostic tests for the diagnosis of nonalcoholic fatty liver disease/nonalcoholic steatohepatitis. World J Gastroenterol 20: 475-485, 2014.

2. Frulio $\mathrm{N}$ and Trillaud H: Ultrasound elastography in liver. Diagn Interv Imaging 94: 515-534, 2013.

3. Itoh A, Ueno E, Tohno E, et al: Breast disease: clinical application of US elastography for diagnosis. Radiology 239: 341-350, 2006.

4. Rago T, Santini F, Scutari M, et al: Elastography: new developments in ultrasound for predicting malignancy in thyroid nodules. J Clin Endocrinol Metab 92: 2917-2922, 2007.

5. Friedrich-Rust M, Ong MF, Herrmann E, et al: Real-time elastography for noninvasive assessment of liver fibrosis in chronic viral hepatitis. AJR Am J Roentgenol 188: 758-764, 2007.

6. Sãftoiu A, Gheonea DI and Ciurea T: Hue histogram analysis of real-time elastography images for noninvasive assessment of liver fibrosis. AJR Am J Roentgenol 189: W232-W233, 2007.

7. Ferraioli G, Gulizia R and Filice C: Real-time elastography in the assessment of liver fibrosis. AJR Am J Roentgenol 189: W170, 2007.

8. Koizumi Y, Hirooka M, Kisaka Y, et al: Liver fibrosis in patients with chronic hepatitis $\mathrm{C}$ : noninvasive diagnosis by means of real-time tissue elastography - establishment of the method for measurement. Radiology 258: 610-617, 2011.

9. Chung JH, Ahn HS, Kim SG, et al: The usefulness of transient elastography, acoustic-radiation-force impulse elastography, and real-time elastography for the evaluation of liver fibrosis. Clin Mol Hepatol 19: 156-164, 2013 
10. Kanamoto M,Shimada M,Ikegami T, et al: Real time elastography for noninvasive diagnosis of liver fibrosis. J Hepatobiliary Pancreat Surg 16: 463-467, 2009.

11. Xie L, Chen X, Guo Q, et al: Real-time elastography for diagnosis of liver fibrosis in chronic hepatitis B. J Ultrasound Med 31 : 1053-1060, 2012.

12. Ochi H, Hirooka M, Koizumi Y, et al: Real-time tissue elastography for evaluation of hepatic fibrosis and portal hypertension in nonalcoholic fatty liver diseases. Hepatology 56: 1271-1278, 2012.

13. Paparo F, Cevasco L, Zefiro D, et al: Diagnostic value of real-time elastography in the assessment of hepatic fibrosis in patients with liver iron overload. Eur J Radiol 82: e755-e761, 2013.

14. Tatsumi C, Kudo M, Ueshima K, et al: Non-invasive evaluation of hepatic fibrosis for type $\mathrm{C}$ chronic hepatitis. Intervirology 53 76-81, 2010.

15. Morikawa H, Fukuda K, Kobayashi S, et al: Real-time tissue elastography as a tool for the noninvasive assessment of liver stiffness in patients with chronic hepatitis C. J Gastroenterol 46: 350-358, 2011.

16. Wang J, Guo L, Shi X, Pan W, Bai Y and Ai H: Real-time elastography with a novel quantitative technology for assessment of liver fibrosis in chronic hepatitis B. Eur J Radiol 81: e31-e36, 2012.

17. Ferraioli G, Tinelli C, Malfitano A, et al: Performance of real-time strain elastography, transient elastography, and aspartate-to-platelet ratio index in the assessment of fibrosis in chronic hepatitis C. AJR Am J Roentgenol 199: 19-25, 2012.
18. Yada N, Kudo M, Morikawa H, et al: Assessment of liver fibrosis with real-time tissue elastography in chronic viral hepatitis. Oncology 84 (Suppl 1): 13-20, 2013

19. Bedossa $P$ and Poynard T: An algorithm for the grading of activity in chronic hepatitis C. The METAVIR Cooperative Study Group. Hepatology 24: 289-293, 1996.

20. Ophir J, Céspedes I, Ponnekanti H, Yazdi Y and Li X: Elastography: A quantitative method for imaging the elasticity of biological tissues. Ultrason Imaging 13: 111-134, 1991.

21. Fiorini E, Cipriano V, De Molo C, et al: Real-time elastography as a noninvasive technique for quantification of fibrosis in patients with chronic viral liver disease: Preliminary findings. J Ultrasound 15: 220-225, 2012.

22. Gheonea DI, Săftoiu A, Ciurea T, et al: Real-time sono-elastography in the diagnosis of diffuse liver diseases. World J Gastroenterol 116: 1720-1726, 2010.

23. Hirooka M, Koizumi Y, Hiasa Y, et al: Hepatic elasticity in patients with ascites: Evaluation with real-time tissue elastography. AJR Am J Roentgenol 196: W766-W771, 2011.

24. Lin SH, Ding H, Mao F, et al: Non-invasive assessment of liver fibrosis in a rat model: Shear wave elasticity imaging versus real-time elastography. Ultrasound Med Biol 39: 1215-1222, 2013. 